\title{
Apolitical Values \& Compliance with Government-Recommended Prosocial Health Behavior
}

\author{
Matt Motta, PhD * \\ Assistant Professor \\ Dept. of Political Science \\ Oklahoma State University \\ Paul Goren, $\mathrm{PhD}$ \\ Professor \\ Dept. of Political Science \\ University of Minnesota
}

At the start of the COVID-19 pandemic, federal government and local governments across the U.S. recommended that individuals engage in social distancing and other prosocial health behaviors (e.g., wear a mask when out in public). While social scientists know a fair amount about the extent to which people complied with these recommendations, far less is known about why some people may have been more likely to do so. Building on insights from Human Values Theory, we argue that people who are more self-transcendent (i.e., more likely to put others' needs before their own) should be more likely to engage in a variety of prosocial health behaviors (PSHB); including social distancing. In a nationally representative survey $(\mathrm{N}=1,015)$ conducted at the start of the coronavirus outbreak, we find that people who are highly selftranscendent were significantly more likely to engage in PSHB. Recognizing the limitations of self-reported data, we validate these findings by merging both international and inter-state phone-tracking data into state and country opinion surveys. We find that, on average, people in both countries and states that place a higher emphasis on self-transcendence values were more likely to engage in social distancing at the start of the pandemic.

Word Count: 3,409

* Corresponding author. (matthew[dot]motta[at]okstate[dot]edu) 
As of this writing, COVID-19 has infected over two million people in the United States. Over 116,000 people have died. ${ }^{1}$ Elected officials and public health officials at the federal, state, and local level have recommended that individuals engage in several prosocial health behaviors (PSHB) - including social distancing, wearing masks when out in public, and avoiding gathering in large groups - to inhibit the spread of the novel coronavirus. While large swaths of the public have complied with these recommendations, many more have not. In this brief note, we ask what factors explain variation in PSHB.

To date, initial research and journalistic commentary have identified tribal political loyalties and objective risk as key factors that affect compliance. Political science research has established that in the realm of political behavior partisans in the mass public follow the lead of political leaders and other co-partisans (Campbell et al. 1960; Goren, Federico, and Kittilson 2009; Lenz 2012). Insofar as President Trump has downplayed the risks posed of the novel coronavirus and failed to follow social distancing guidelines, it is unsurprising to see evidence that partisan affinities shape PSHB. Republicans are less likely to take preventative measures than Democrats are, and the president's supporters are less likely to comply with public health recommendations than are his opponents. ${ }^{2}$ In addition, the objective risk of contracting and dying from COVID-19 varies widely across population subgroups. The elderly, people who suffer from underlying medical conditions (e.g., diabetes) and people of color have proven especially vulnerable to the disease. To minimize their risk, members in these groups may be more likely to comply with social distancing recommendations.

The fact that partisan affinities correlate with disease exposure further complicates matters. As of June 2020, COVID 19 death toll is much higher in places where Democrats live.

\footnotetext{
${ }^{1}$ https://coronavirus.jhu.edu/map.html. Accessed June 16, 2020.

2 See this working paper from Alcott and colleagues (http://web.stanford.edu/ gentzkow/research/social_distancing.pdf) as well as this recent poll from Gallup (https://news.gallup.com/opinion/polling-matters/311087/partisan-gap-views-coronavirus.aspx).
} 
As reported by the Pew Research Center, the COVID 19 death toll is heavily concentrated in Democratic congressional districts, ${ }^{3}$ while Reuters reports that death rates in U.S. counties that voted for Hillary Clinton in 2016 are three times higher than counties that voted for Donald Trump. ${ }^{4}$ Hence, the propensity for Republicans/Trump supporters to undertake less social distancing than Democrats/Trump opponents could at least in part result from living in communities less directly impacted by the coronavirus.

While tribal political loyalties and objective risk provide plausible explanations for variation in PSHB, such explanations are surely incomplete. In this brief note, we examine the degree to which PSHB correlates with a pair of basic human values that shape individual behavior on a daily basis. Basic human values are cognitive representations of desirable endstates or modes of conduct that vary in terms of personal importance, transcend specific situations, and guide behavior (Rokeach 1973; Schwartz 1994). In the leading values theory, Shalom Schwartz $(1994,2012)$ posits that four superordinate values exist in the minds of people across all cultures and societies. This model has been validated by researchers across the psychological sciences in hundreds of studies over the past 40 years (Schwartz and Boehnke 2004; Spini 2003).

Two values seem especially likely to shape PSHB. First, self-transcendence values stress acceptance of and concern for the welfare of other people, such as in-groups members (e.g., parents, siblings, friends, etc.) and out-groups (e.g., community members, vulnerable populations, the world community, etc.). By prioritizing the well-being of others, we can think of self-transcendence as pro-social values. We propose that the more importance people attach to self-transcendence, the more social distancing behavior they will undertake. Second, self-

\footnotetext{
${ }^{3}$ https://www.pewresearch.org/fact-tank/2020/05/26/coronavirus-death-toll-is-heavily-concentrated-in-democratic-congressional-districts/. Accessed June 16, 2020

${ }^{4}$ https://www.reuters.com/article/us-health-coronavirus-usa-divided/divided-by-covid-19-democratic-u-s-areas-hit-three-times-as-hard-asrepublican-ones-idUSKBN22X14I
} 
enhancement values prioritize the pursuit of self-interest, personal fulfillment, and success at the expense of others (Schwartz 1994, 2003). By prioritizing self-interest, we should think of selfenhancement as pro-individual values. We predict that the more importance people attach to selfenhancement, the less PSHB they will undertake.

While tribal partisan loyalties and objective risk surely matter, we posit that personal values assume a critical role in deciding whether to social distance. Since people have a lot of experience linking values to personal choice in their day-to-day lives (Fischer and Smith 2004; Homer and Kahle 1988; Maio and Olson 1995; Verplanken and Holland 2002; Zibenberg and Kupermintz 2016), the extension to PSHB seems straightforward. This is important because it suggests that PSHB represents far more than tribal political mimicry and risk assessments. The degree to which people prioritize their own well-being and the well-being of others should matter as well.

In what follows, we assess these expectations by first testing for an association between self-transcendance (ST) and self-enhancement (SE) values, and several forms of PSHB in a cross-sectional survey of U.S. adults; controlling for factors such as partisan identification and county-geocoded COVID-19 disease prevalence that could alternatively shape PSHB. We then validate these results by merging aggregated phone-tracking social distancing data into large public opinion surveys which measured ST values at both the state and country level. Both the individual-level survey results and aggregate-level phone tracking validation exercise provide strong support for our theoretical expectations.

\section{Survey Data \& Measures}

Data. 
Survey data for this study come from a demographically representative sample of U.S. adults (N $=1,015)$ conducted on April 26, 2020. Note that we surveyed respondents in advance of the first states (Oklahoma and Georgia) lifting "stay at home" orders and easing social distancing restrictions on May 1, 2020.

Respondents were invited to participate in the survey via Lucid Theorem's large online opt-in sample, which employed quota sampling procedures to ensure demographic representativeness on several factors: including respondent's age, race, gender, partisan identification, educational attainment, household income, Census region.

We apply post-stratification weights on the basis of age, race, educational attainment, income, and gender to account for any remaining differences between the sample and target population. As Table S1 in the Supplementary Materials suggests, both the weighted and unweighted sample data closely reflect nationally representative demographic benchmarks.

\section{Measures \& Analytical Procedure.}

We test our theoretical expectations by regressing a multi-item, self-reported indicator of Pro-Social Health Behavior (PSHB) on Self-Transcendence (ST) and Self-Enhancement (SE) values. We measure $P S H B$ by asking respondents whether or not (via a multiple-answer checkbox item) they "in the past month" took any of nine behavioral actions "as a result of the novel coronavirus outbreak;" including wearing a mask when out in public, avoiding dining-in at restaurants or bars, and leaving the house only to participate in essential activities like grocery shopping. We provide a full list in the Supplementary Materials.

We combined items into a single index $(\mathrm{M}=0.62, \mathrm{SD}=0.29)$ using a two parameter logistic application of item response theory. This approach is advantageous - as opposed to 
summing up the items - because it allows each indicator to contribute differently to where respondents are placed on a latent behavioral continuum. Additional diagnostic information about this procedure can be found in the Supplement.

We then measured $S T$ and $S E$ using standard multiple-item indicators that asked whether respondents agree or disagree (on a seven point Likert scale) with five items designed to tap benevolence and universalistic values (ST; e.g., "It's very important to me to help the people around me. I want to care for other people") and four items designed to tap achievement and power values (SE; e.g., "It is very important to show others my abilities. I want people to admire what I do.”) Consistent with conventional practice (e.g., Goren et al., 2020), we compute average scores on both the ST $(a=0.87)$ and SE $(a=0.87)$ items. A full list of items can be found in the Supplementary Materials.

Our models control for several factors that could alternatively explain why some people choose to participate in PSHB; including Political Ideology (a standard seven-point ideological self-placement scale; scored so that higher values indicate strong conservatism), Partisan Identification (dichotomous indicators of whether or not people self-identify as Democrats or Republicans), POTUS Trust (a four-category indicator of whether or not respondents feel that they can trust the president to "do what is right" - ranging from "do not trust at all" to "trust greatly"), Anti-Expert Attitudes (a seven-point scale denoting agreement with the statement that respondents would "rather place trust in the wisdom of ordinary people than the opinions of experts and intellectuals;" see: Oliver \& Rahn 2016; Motta 2018; Merkley 2020), and COVIDRelated Misinformation Endorsement (binary indicators of whether or not respondents believe that COVID-19 was manufactured in a lab, or that a vaccine existed as of April 2020; see: Motta et al., 2020). All factors were re-coded to range from 0-1. 
We also control for binary indicators of respondents' Age (age cohort indicators), Race (whether respondents are Black [Non-Hispanic], or Hispanic), Educational Attainment (whether or not they completed college), and Gender (i.e., whether respondents self-identify as women). Finally, we control for Per Capita COVID-19 Prevalence by merging publicly available data from Johns Hopkins CSSEGIS COVID-19 tracking database ${ }^{5}$ into the opinion data; aggregated across counties from 4/1-4/26 (to match the frame of reference in the PSHB items), calculated at the (county) per capita level, and re-scaled to range from 0-1. Technical information about our geocoding procedures can be found in the Supplementary Materials.

\section{Results}

Figure 1 presents the results of an OLS model regressing PSHB on ST and SE values. Parameter estimates (arranged in descending order, by effect size) are presented as shaded circles, with 95\% confidence intervals extending out from each one. Confidence intervals that do not intersect with the dashed line indicate that their associated parameter estimate is significantly associated with PSHB at the $\mathrm{p}<0.05$ level, two-tailed.

Full model output is available in the Supplementary Materials. Note that because all variables are scored to range from $0-1$, parameter estimates can be interpreted as the percent change in PSHB, given movement from the minimum to maximum value of each variable. Consistent with our expectations, we find that ST values are both strongly $(\mathrm{B}=0.37)$ and significantly ( $\mathrm{p}<0.05$, two-tailed) associated with increased PSHB. Conversely, and again consistent with expectations, we find that SE values are associated with a statistically significant decrease $(\mathrm{B}=-0.18, \mathrm{p}<0.05)$ in self-reported PSHB. Although the (positive) effects of ST are

\footnotetext{
5https://github.com/CSSEGISandData/COVID19/tree/master/csse_covid_19_data/csse_covid_19_time_series
} 
somewhat larger than the (negative) effects of SE, both factors exhibit the strongest positive and negative relationships with PSHB (respectively).

In addition to the effects of basic human values, we find that older, college-educated, and respondents living in counties where COVID-19 cases were comparatively more prevalent were significantly more likely to engage in PSHB. Respondents who hold negative views toward scientific experts, and those who endorse misinformation about a potential COVID-19 vaccine, were less likely to self-report engaging in PSHB. Interestingly, and consistent with other selfreported behavioral research conducted in April 2020, ${ }^{6}$ we find little evidence of partisan or ideological asymmetries in PSHB.

\section{Figure 1. The Effect of ST \& SE Values on Self-Reported PSHB (April 2020 Survey)}

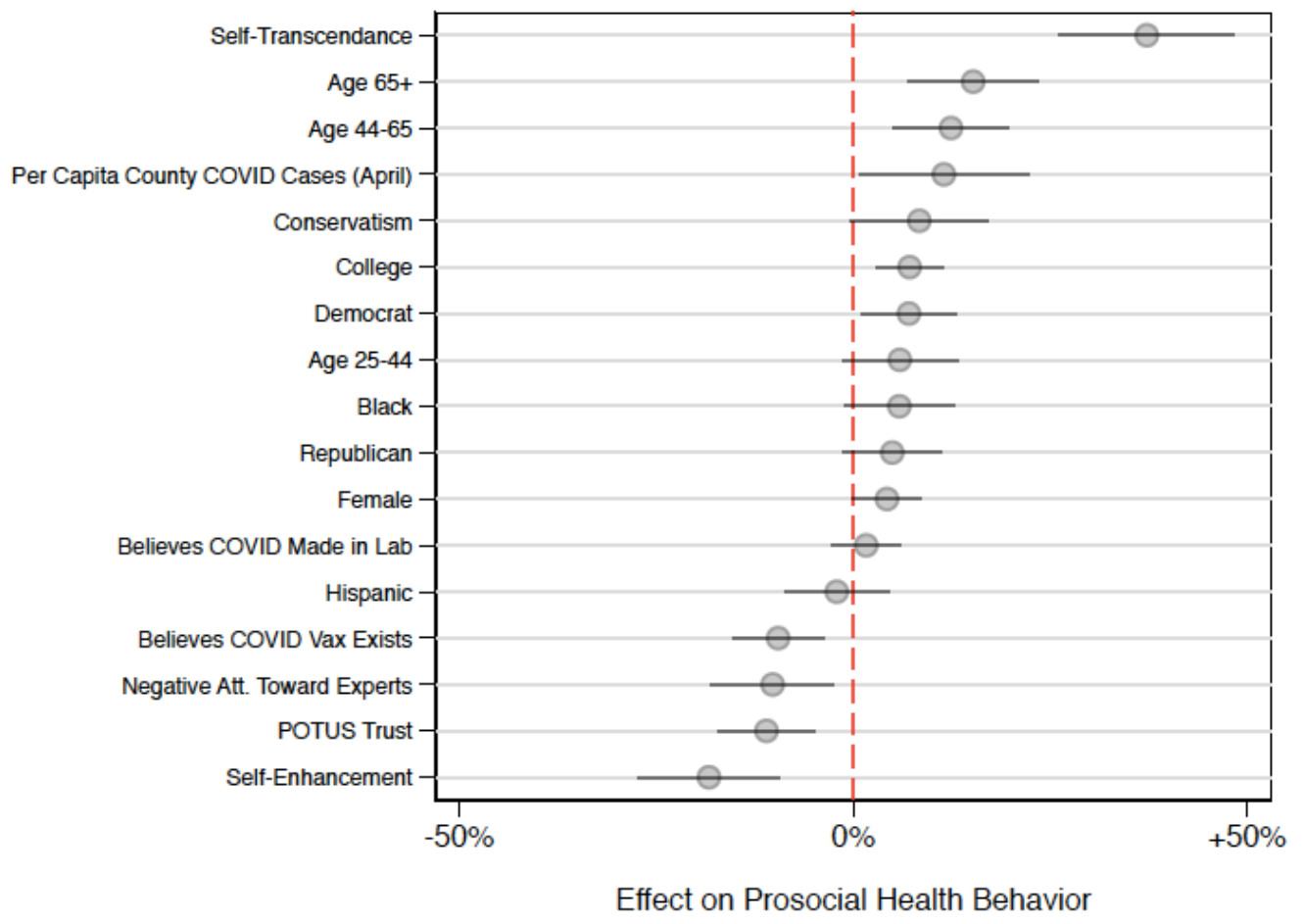

OLS Regression Parameter Estimates w/95\% Confidence Intervals

Note. $N=1,1015$. OLS parameter estimates with 95\% confidence intervals presented. Please refer to the Supplemental Materials for full model output. Post-stratification weights are applied.

\footnotetext{
${ }^{6}$ https://today.yougov.com/topics/politics/articles-reports/2020/04/16/coronavirus-social-distancing-americans-not-so-div
} 


\section{Validation with Geolocated Social Distancing Data}

Our survey data provide strong evidence that ST is positively correlated with PSHB, and more modest evidence that SE is negatively correlated with it. However, as we measure PSHB via self-reports, some might ask whether or not our results are influenced social desirability bias (e.g., Chang \& Krosnick 2009). For example, it could be the case that highly ST individuals are more likely to say that they participate in PSHB without actually doing so.

Consequently, we test the robustness of our findings by assessing whether or not variation in average state and country-level indicators of ST values are positively associated with measures of actual social distancing behavior; measured via publicly available phone-tracking data from Google. ${ }^{7}$

We created a state-level indicator of ST by calculating average universalism value scores (as a proxy for ST more generally) across states from a nationally representative YouGov survey of $\mathrm{N}=10,000$ U.S. adults interviewed in 2011 (see: Goren et al., 2020). Similarly, we created a cross-national ST indicator based on average country-level benevolence and universalism value scores, from 60 countries sampled in Wave IV the World Values Survey (WVS; see: WVS 2020). Note that we limit our validation exercise to just ST values due to data constraints in the state-level data (as SE values were not administered), and because ST values exhibit the clearest relationship with PSHB in the survey data.

We then matched Google's state and country-level estimates (respectively) of average reduction in distance traveled to non-residential places - including parks, retail stores, grocery/pharmacies, transit stations, and workplaces - compiled between 2/15 - 4/17, 2020 . Google tracked these figures by comparing mobile phone users' daily movement to-and-from different geotagged locations (pursuant with the categories listed above) in Spring 2020; in

\footnotetext{
${ }^{7} \mathrm{https} / / /$ www.google.com/covid19/mobility/data_documentation.html?hl=en
} 
comparison to baseline figures calculated from 1/3-2/6, 2020. Google has made these data publicly available. We note that while Google tracking data was available for all fifty states (and D.C.), 11 countries (e.g., China) included in WVS did not have tracking data available. We omit those countries from analysis.

Figure 2 presents the results of five scatterplots - with lines of best fit expressed as dashed lines - assessing the correlation between variation in state-level ST and average change in the distance traveled to each of the previously mentioned non-residential places. Across the states, we find a moderate and negative correlation between ST values and increased travel; with correlations ranging from a minimum of $r=-0.22$ (retail travel) to $r=-0.39$ (parks travel). This means that people in states with higher than average ST values were more likely to engage in social distancing behavior; lending additional support for our theoretical expectations.

In Figure 3, we uncover an analogous pattern in the cross-national data. Again, we find a moderately strong (and negative) correlation between distance traveled and ST values; ranging from a minimum of $r=-0.25$ (workplaces) to -0.51 (parks). This again lends validation to the survey results presented earlier, suggesting that people in countries with higher than average ST values were more likely to engage in social distancing behavior.

\section{Conclusions \& Discussion}

Our study shows that basic human values are strongly associated with prosocial health behavior (PSHB). Consistent with our expectations, people who place a higher emphasis on selftranscendence (ST) values are more likely to engage in PSHB, while those who place a higher emphasis on self-enhancement (SE) values are not. Recognizing the limits of self-reported behavioral data, we validate these results using geolocated phone-tracking indicators of social 
distancing behavior. People living in both states and countries that place higher (vs. lower) emphasis on self-transcendence values are more likely to engage in social distancing.

These results suggest that basic and apolitical value orientations play an important role in shaping public health behavior, and can help explain why some people were more likely to follow government-recommended isolation and distancing procedures than others.

Of course, this study is limited in that it investigates the correlates of health behavior at just a single moment in time. Scholars might consider longitudinally investigating how ST and SE affect change in PSHB, in later stages of the pandemic; especially in response to changing state and federal guidelines. It could be the case, for example, that individuals who strongly value ST are more likely to practice social distancing behavior, even in the absence of government recommendations and mandated closures of non-residential places. We welcome and look forward to future research in this area. 
Figure 2. The Effect of ST Values on State-Level Social Distancing Behavior (Phone Tracking Data)
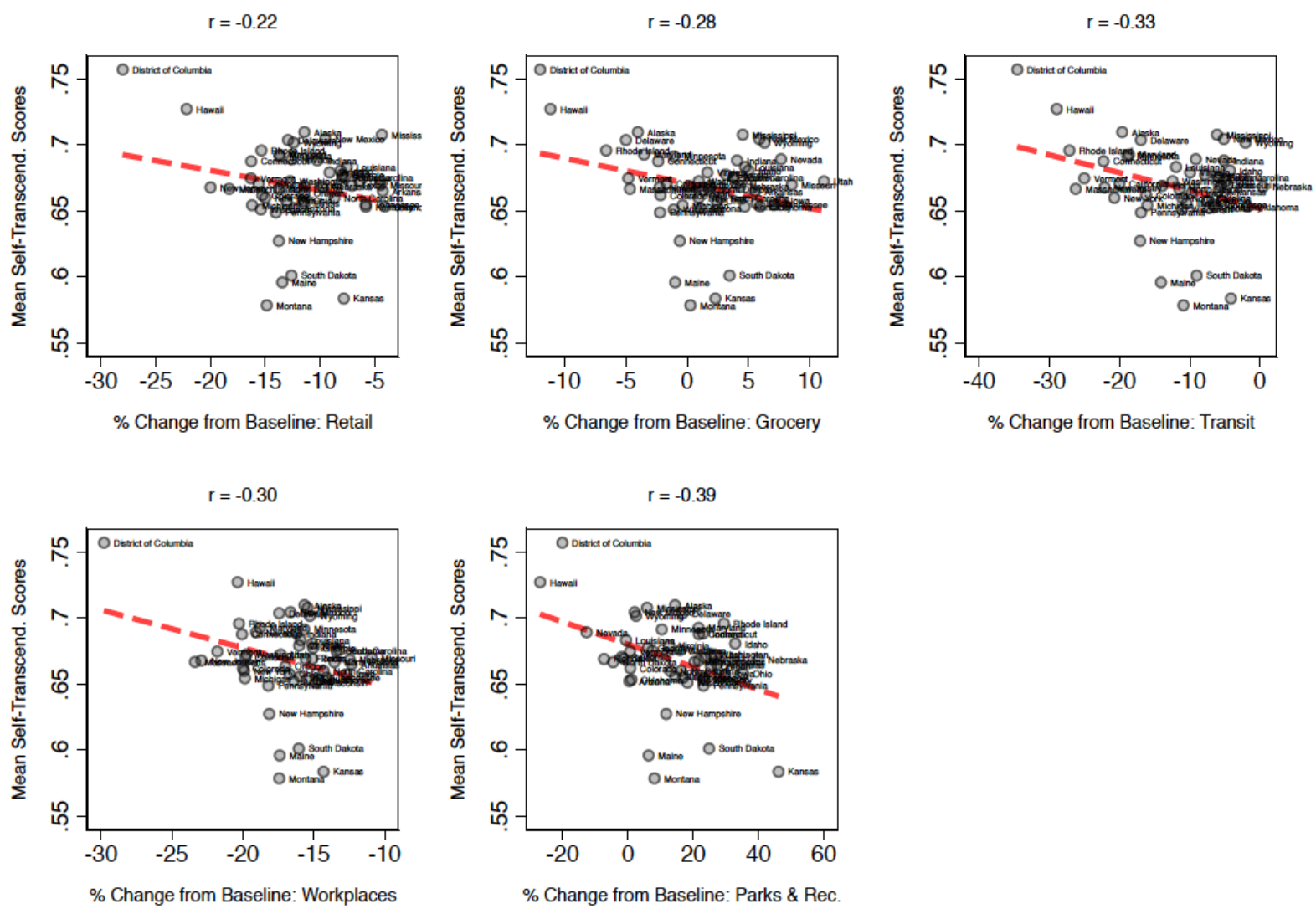

Note. Scatterplot with a line of best fit presented. State-level data are derived from a nationally representative 2011 YouGov survey of $N=10,000$ U.S. Adults, and matched to phone-tracking data from Google (comparing average daily movement from 2/15-4/17, 2020 to baseline daily estimates from 1/3-2/6, 2020). 
Figure 3. The Effect of ST Values on Country-Level Social Distancing Behavior (Phone Tracking Data)
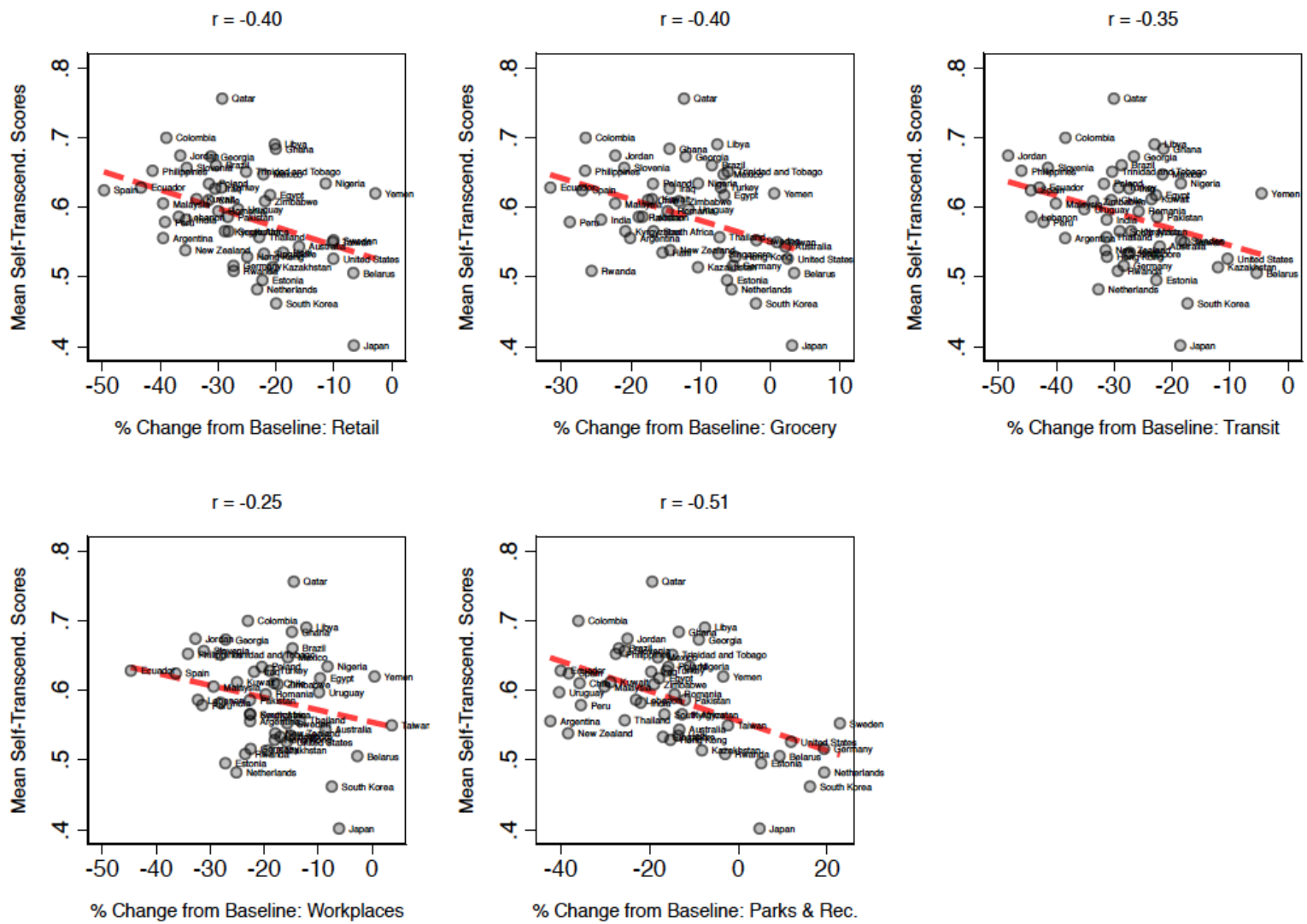

Note. Scatterplot with a line of best fit presented. State-level data are derived from a nationally representative 2011 YouGov survey of N=10,000 U.S. Adults, and matched to phone-tracking data from Google (comparing average daily movement from 2/15-4/17, 2020 to baseline daily estimates from 1/3-2/6, 2020). 


\section{References}

Campbell, Angus, et al. The American Voter. University of Chicago Press, 1980.

Chang, L., \& Krosnick, J. A. (2009). National surveys via RDD telephone interviewing versus the Internet: Comparing sample representativeness and response quality. Public Opinion Quarterly, 73(4), 641-678.

Fischer, R., \& Smith, P. B. (2004). Values and organizational justice: Performance-and senioritybased allocation criteria in the United Kingdom and Germany. Journal of Cross-Cultural Psychology, 35(6), 669-688.

Goren, P., Federico, C. M., \& Kittilson, M. C. (2009). Source cues, partisan identities, and political value expression. American Journal of Political Science, 53(4), 805-820.

Goren, P., Smith, B., \& Motta, M. (2020). Human Values and Sophistication Interaction Theory. Political Behavior.

Homer, P. M., \& Kahle, L. R. (1988). A structural equation test of the value-attitude-behavior hierarchy. Journal of Personality and social Psychology, 54(4), 638.

Lenz, G. S. (2013). Follow the leader?: how voters respond to politicians' policies and performance. University of Chicago Press.

Maio, G. R., \& Olson, J. M. (1995). Relations between values, attitudes, and behavioral intentions: The moderating role of attitude function. Journal of experimental social psychology, 31(3), 266-285.

Merkley, E. (2019). Anti-Intellectualism, Populism, and Motivated Resistance to Expert Consensus. Forthcoming at Public Opinion Quarterly.

Motta, M. (2018). The dynamics and political implications of anti-intellectualism in the United States. American Politics Research, 46(3), 465-498.

Motta, M., Stecula, D., \& Farhart, C. (2020). How Right-Leaning Media Coverage of COVID-19 Facilitated the Spread of Misinformation in the Early Stages of the Pandemic in the US. Canadian Journal of Political Science/Revue canadienne de science politique, 1-8. 
Oliver, J. E., \& Rahn, W. M. (2016). Rise of the Trumpenvolk: Populism in the 2016 Election. The ANNALS of the American Academy of Political and Social Science, 667(1), 189-206.

Rokeach, M. (1973). The nature of human values. Free press.

Schwartz, S. H. (1994). Are there universal aspects in the structure and contents of human values?. Journal of social issues, 50(4), 19-45.

Schwartz, S. H. (2003). A proposal for measuring value orientations across nations. Questionnaire package of the european social survey, 259(290), 261.

Schwartz, S. (2012). Toward refining the theory of basic human values. In Methods, theories, and empirical applications in the social sciences (pp. 39-46). VS Verlag für Sozialwissenschaften.

Schwartz, S. H., \& Boehnke, K. (2004). Evaluating the structure of human values with confirmatory factor analysis. Journal of research in personality, 38(3), 230-255.

Spini, D. (2003). Measurement equivalence of 10 value types from the Schwartz value survey across 21 countries. Journal of cross-cultural psychology, 34(1), 3-23.

Verplanken, B., \& Holland, R. W. (2002). Motivated decision making: effects of activation and self-centrality of values on choices and behavior. Journal of personality and social psychology, 82(3), 434. 